\title{
COMPARACIÓN DE LA RELACIÓN DE FENOLES TOTALES, FLAVONOIDES Y CAPACIDAD ANTIOXIDANTE EN BRÁCTEAS DE DOS VARIEDADES DE BOUGAINVILLEA GLABRA CHOISY
}

\author{
COMPARISON OF TOTAL PHENOLS, FLAVONOIDS \\ AND ANTIOXIDANT CAPACITY RELATIONSHIP IN BRACTS \\ OF TWO VARIETIES OF BOUGAINVILLEA GLABRA CHOISY
}

\section{Carmita Jaramillo J. ${ }^{1}$, Juan C. Armijos A. ${ }^{1}$, Raquel Cedeño S. ${ }^{1}$, Mercedes Campo F. ${ }^{1}$ \& Luisa Rojas $^{2 *}$}

Recibido: 13 de noviembre 2020 / Aceptado: 17 de diciembre 2020 DOI: 10.26807/ia.v9i1.203

Palabras clave: Brácteas, colores, DPPH; extracto seco, B. glabra. Keywords: Bract colors, DPPH', dry extract, B. glabra.

\section{RESUMEN}

Con el objetivo de comparar la relación existente entre el contenido de fenoles totales, flavonoides y la capacidad antioxidante en las brácteas naranjas y moradas de Bougainvillea glabra Choisy, se obtuvieron extractos secos (ES) a partir de muestras de brácteas secas y molidas utilizando como disolvente una mezcla

1 Universidad Técnica de Machala, Machala-Ecuador. (cjaramillo@utmachala.edu.ec., jcarmijosa 1994_est@utmachala.edu.ec, ecedeno_est@utmachala.edu.ec, mcampo@utmachala.edu.ec. 2 Universidad de Oriente, Cumana-Venezuela ( ${ }^{*}$ correspondencia: Irojas40@yahoo.com) 
hidroalcohólica (50 \%) y sonicación. Los contenidos promedios de fenoles totales (mg de EAG/g ES) y de flavonoides (mg EQ/g ES) resultaron ser los más altos en la variedad naranja. Se evaluó la capacidad antioxidante usando el método de la inhibición del radical libre 2,2-difenil-1-picrilhidrazilo (DPPH·), obteniéndose que las brácteas naranjas presentaron la mayor capacidad antioxidante, con una concentración inhibitoria del $50 \%\left(\mathrm{Cl}_{50}\right)$ promedio de 37 $\mu \mathrm{g} / \mathrm{mL}$, mientras que en la variedad morada el $\mathrm{Cl}_{50}$ fue de $270 \mu \mathrm{g} / \mathrm{mL}$. La correlación entre fenoles y flavonoides resultó ser altamente positiva, mientras que entre estos compuestos químicos y la capacidad antioxidante fue altamente negativa. Ambas muestras de B. glabra exhiben potencial antioxidante, siendo la superior en las brácteas de color naranja, y con relaciones significativas positivas $(p<0,05)$ con los niveles de fenoles totales y flavonoides.

\section{ABSTRACT}

To compare the relationship between the content of total phenols, flavonoids and the antioxidant capacity in the orange and purple bracts of Bougainvillea glabra Choisy, dry extracts (DE) were obtained from dried and ground samples of bracts, using as solvent a hydroalcoholic mixture (50 \%) and sonication. The average contents of total phenols (mg EAG/g DE) and flavonoids (mg EQ/g DE) were the highest in the orange variety. The antioxidant capacity was evaluated using the 2,2-diphenyl-1-picrylhydrazyl (DPPH) free radical inhibition method, obtaining that the orange bracts presented the highest antioxidant capacity, with an inhibitory concentration of $50 \%\left(\mathrm{IC}_{50}\right)$ of $37 \mu \mathrm{g} / \mathrm{mL}$, while that in the purple variety the $\mathrm{IC}_{50}$ was $270 \mu \mathrm{g} / \mathrm{mL}$. The correlation between phenols and flavonoids was highly positive, while between these chemical compounds and the antioxidant capacity were highly negative. Both samples of $B$. glabra exhibited antioxidant capacity, being the highest in orange bracts, and with significant positive relationships $(\mathrm{p}<0.05)$ with the levels of total phenols and flavonoids. 


\section{INTRODUCCIÓN}

Durante las últimas dos décadas se ha informado, mediante evidencias, de la implicación de los radicales libres en una amplia variedad de enfermedades humanas (Jamshidi-Kia et al., 2020). En las células humanas, la principal molécula generadora de radicales libres es la del oxígeno, el cual al entrar al organismo interviene en una serie de reacciones de oxidoreducción complejas en las que se producen algunas especies derivadas de este que son reactivas, denominadas especies reactivas del oxígeno (ERO). Si la generación de ERO es alta y supera la eficacia de la defensa antioxidante del organismo surge una condición Ilamada estrés oxidativo (Reyes et al., 2011), que puede conducir a daños en las células, mutación de los genes y carcinogénesis (Alkadi \& Ifeany 2018; Jamshidi-Kia et al., 2020) y provocar la incidencia de diversas enfermedades, entre ellas la esclerosis lateral amiotrófica, las enfermedades de Parkinson y Alzheimer (Cenini et al., 2019). Por lo que se hace necesario el consumo de compuestos antioxidantes para retardar o inhibir la oxidación de sustratos susceptibles a las ERO (Reyes et al. 2011; Coronado et al., 2015).
Las investigaciones en la química de productos naturales, en la búsqueda de compuestos eficaces y no tóxicos con actividad antioxidante, se ha convertido en una tendencia en los últimos años (Rao et al., 2015; Jamshidi-Kia et al., 2020).

Adicionalmente, la estrategia de la Organización Mundial de la Salud (para el período 2014-2023) (WHO, 2013), promueve la utilización de la medicina tradicional y complementaria (T\&CM) mediante políticas que permitan a las personas acceder a la T\&CM de manera segura y eficaz, tomando como base la investigación científica para la incorporación de productos naturales en los sistemas de salud que cumplan con los estándares de calidad establecidos.

La especie vegetal Bougainvillea glabra Choisy es endémica de América del Sur y está muy extendida en todo el mundo (Abarca \& Petricevich, 2018). En la medicina tradicional, a las brácteas se le han atribuido varias acciones, entre ellas su efectividad en el tratamiento de enfermedades respiratorias (asma) (Alonso-Castro et al., 2017), capacidad antibacteriana 
(Enciso-Díaz et al., 2012), y actividad antioxidante (Naidu et al., 2016; Abarca \& Petricevich, 2018).

Los análisis fitoquímicos de diferentes partes de B. glabra Choisy han dado a conocer que contiene 105 diferentes compuestos químicos, los cuales pertenecen a las siguientes familias de productos naturales: alcaloides, taninos, fenoles, flavonoides, betacianinas, terpenoides, glucósidos y aceites esenciales (Heuer et al., 1994; Saleem et al., 2021).
Aunque B. glabra se reporta dentro de las plantas que presentan actividad antioxidante, dicha propiedad biológica no está definida en las brácteas de diferentes colores de la especie. Por tal motivo, se determinó la relación existente entre el contenido de fenoles totales, flavonoides y la capacidad antioxidante de las brácteas de $B$. glabra de color naranja y morada.

\section{MATERIALES Y MÉTODOS}

Se recolectaron muestras representativas de las brácteas de $B$. glabra, de colores naranja y morada, en las localidades de Machala ( $3^{\circ} 28^{\prime} 60,64^{\prime \prime}$

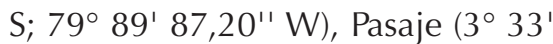
$89,07^{\prime \prime} \mathrm{S} ; 79^{\circ} 81^{\prime} 45,99^{\prime \prime} \mathrm{W}$ ) y Piñas $\left(3^{\circ} 68^{\prime} 73,03^{\prime \prime} \mathrm{S} ; 79^{\circ} 61^{\prime} \mathrm{O}\right.$ 09,05' 'W) de la provincia de El Oro, Ecuador. Luego, se sometieron al proceso de lavado y secado a temperatura ambiente por 48 horas; posteriormente, se colocaron en una estufa (SNB400, Memmert), por 48 horas a $40 \pm 2{ }^{\circ} \mathrm{C}$. Finalmente, se molieron hasta obtener un polvo fino de tamaño de partícula $\leq 1 \mathrm{~mm}$.

\section{Preparación de los extractos secos}

$10 \mathrm{~g}$ de las muestras secas y molidas de las brácteas de $B$. glabra, naranjas y moradas, se mezclaron con $100 \mathrm{~mL}$ de etanol (50\%) y se sometieron a sonicación, utilizando un baño ultrasónico (ULTRASONIC BATH 5,7 L, Fischer Scientific). Posteriormente, los extractos se filtraron y se concentraron hasta sequedad en un rotoevaporador (HEIDOLPH LABOROTA 4001 efficient) acoplado a un criostato (LAUDA/ALPHA RA-8) y a una bomba de vacío (VACUUBRAND PC 600 , Alemania). Finalmente, se alma- 
cenaron los extractos secos a $4^{\circ} \mathrm{C}$ y protegidos de la luz hasta su posterior análisis.

\section{Cuantificación del contenido de fe- noles}

La cuantificación de fenoles se realizó por el método de Folin-Ciocalteu. Para la curva de calibración se partió de una disolución de ácido gálico (EAG) (Sigma Aldrich) como estándar, a una concentración de 10 $\mathrm{mg} / \mathrm{mL}$, para preparar seis disoluciones patrón de concentraciones 0,1 $0,9 \mathrm{mg} / \mathrm{mL}$. De cada dilución se tomaron $20 \mu \mathrm{L}$, a los cuales se le añadió $1 \mathrm{~mL}$ de disolución del reactivo Folin-Ciocalteu (Sigma-Aldrich, Alemania) $(0,2 \mathrm{~mol} / \mathrm{L})$ y $180 \mu \mathrm{L}$ de agua destilada. Se agitó y se esperaron 5 minutos. Se adicionaron, por último, $800 \mu \mathrm{L}$ de carbonato de sodio al 7,5 $\%$, se agitó y se dejó reposar por 2 horas. Se midieron las absorbancias a las disoluciones de ácido gálico en un espectrofotómetro (Evolution 201, Thermo Scientific), a 765 nm. Se utilizó un blanco que se preparó bajo las mismas condiciones. El ensayo se realizó por triplicado. Se preparó una disolución al 1 \% del extracto seco (ES) en metanol, de allí se tomaron
$20 \mu \mathrm{L}$ y se procedió de la misma manera antes descrita. Los datos se expresaron como miligramos equivalentes de ácido gálico por gramo de ES (mg de EAG/g ES).

\section{Cuantificación de flavonoides}

Se siguió la metodología propuesta por Lin \& Tang (2007), con algunas modificaciones. Se utilizó como estándar quercetina de 1 mg/mL (Sigma Aldrich) y desde allí se prepararon diluciones en el rango (25 y $125 \mu \mathrm{g} / \mathrm{mL}$ ) para la curva de calibración, por triplicado. Se transfirieron $0,5 \mathrm{~mL}$ de cada disolución de quercetina y se mezcló con 1,5 mL de etanol $95 \%$ a un tubo de ensayo de capacidad de 5 $\mathrm{mL}$. Luego, se añadió $0,1 \mathrm{~mL}$ de disolución acuosa de cloruro de aluminio $\left(\mathrm{AlCl}_{3}\right)$ al $10 \%, 0,1 \mathrm{~mL}$ de acetato de potasio $1 \mathrm{~mol} / \mathrm{L}$ y $2,8 \mathrm{~mL}$ de agua destilada. Se dejó reposar a temperatura ambiente durante 40 minutos, para luego proceder a la lectura en el espectrofotómetro, estableciendo una longitud de onda de $415 \mathrm{~nm}$, frente al blanco que se preparó bajo las mismas condiciones de los patrones. Para el ensayo se prepararon disoluciones acuosas $(1 \% \mathrm{~m} / \mathrm{v})$ de los ES de las muestras naranja y morada, respecti- 
vamente. De allí se tomaron $0,5 \mathrm{~mL}$ y se procedió de la misma manera antes descrita para la curva de calibración. El contenido total de flavonoides se determinó por triplicado y los datos se expresaron como miligramos equivalentes de quercetina (mg EQ/g de ES).

\section{Determinación de la actividad antio- xidante}

La actividad antioxidante se evaluó usando el radical libre 2,2-difenil-1picrilhidrazilo (DPPH $)$. Se siguió la metodología de Brand-Williams et al. (1995), con modificaciones. Para ello se preparó una disolución de DPPH. en metanol 0,1 $\mathrm{mmol} / \mathrm{L}$ protegido de la luz. Se prepararon disoluciones acuosas al 1\% $(\mathrm{m} / \mathrm{v})$ de ambos ES y se diluyeron a varias concentraciones $(0,05 ; 0,1 ; 0,2 ; 0,3 ; 0,4 ; 0,5) \mathrm{mg} / \mathrm{mL}$. Luego, se transfirió $0,5 \mathrm{~mL}$ de cada solución por separado en un tubo de ensayo y se agregaron 1,5 mL de la solución metanólica de $\mathrm{DPPH}$, se agitó y almacenó en la oscuridad por 30 minutos. Se consideró un grupo control por cada réplica, utilizando la disolución de DPPH, para proceder a la lectura en un espectrofotómetro, estableciendo una longitud de onda $516 \mathrm{~nm}$. El ensayo se realizó por triplicado y el porcentaje secuestro del radical libre DPPH. se determinó mediante la siguiente fórmula:

$\% \mathrm{DPPH}=\frac{\text { Absorbancia (control) }- \text { Absorbancia (muestra) }}{\text { Absorbancia (muestra) }} \times 100$

Con los porcentajes de secuestro de $\mathrm{DPPH}$ - obtenidos y las concentraciones de cada solución de muestra ensayada, se obtuvo por regresión lineal una ecuación que permitió obtener la concentración media inhibitoria $\left(\mathrm{Cl}_{50}\right)$.

\section{Análisis estadístico}

Todos los resultados fueron analizados usando el programa estadístico STATGRAPHICS CENTURION XVI.II para realizar la prueba ANOVA $(p<0,05)$ para muestras independientes y el coeficiente de correlación de Pearson para relacionar el contenido de fenoles totales, flavonoides y la capacidad antioxidante de las brácteas de B. glabra. 


\section{RESULTADOS}

Los resultados de la cuantificación de fenoles por el método espectrofotométrico de Folin-Ciocalteu muestran que se obtuvo una linealidad aceptable, con un $R^{2}=0,996$ en la ecuación de la recta. La concentración promedio de fenoles en las brácteas naranjas fue 129,6 \pm 0,3 mg EAG/g de ES y en las brácteas moradas $79,9 \pm$ $0,7 \mathrm{mg} \mathrm{EAG/g}$ de ES (Tabla 1). Se constataron diferencias estadísticamente significativas $(p<0,05)$, existiendo mayor contenido de fenoles totales en las brácteas naranjas, en comparación con las brácteas moradas.

Para la determinación cuantitativa de flavonoides se obtuvo una curva de calibración con linealidad aceptable $\left(R^{2}=0,99\right)$ de la ecuación de la recta. En la Tabla 1 se pueden observar los resultados de flavonoides obtenidos para las brácteas de $B$. glabra. Para las brácteas naranjas fueron 40,1 $\pm 0,2$ mg EQ/g ES y para las brácteas moradas 9,1 $\pm 0,3 \mathrm{mg} \mathrm{EQ/g} \mathrm{ES.} \mathrm{Existe} \mathrm{una}$ diferencia estadísticamente significativa $(p<0,05)$ en el contenido flavonoides, siendo mayor en las brácteas naranjas, en comparación con las de color morado.
Las disoluciones del extracto seco de las brácteas naranjas lograron inhibir el $50 \%$ del radical libre a una menor concentración (37 $\mu \mathrm{g} / \mathrm{mL}$ ) que las brácteas moradas (270 $\mu \mathrm{g} / \mathrm{mL})$ (Tabla 1$)$.

Tabla 1. Concentraciones de fenoles (mg EAG/g ES), flavonoides (mg EQ/g ES) y coeficiente de inhibición medio $\left(\mathrm{Cl}_{50}, \mu \mathrm{g} / \mathrm{mL}\right)$ en brácteas naranjas y moradas de B. glabra

\begin{tabular}{cccc}
\hline Muestras & Fenoles & Flavonoides & $\mathrm{Cl}_{50}$ \\
\hline Naranja & $129,6 \pm 0,3$ & $40,1 \pm 0,2$ & $37 \pm 2$ \\
Morada & $79,9 \pm 0,7$ & $9,1 \pm 0,3$ & $279 \pm 4$ \\
\hline
\end{tabular}

Al correlacionar la cantidad de fenoles totales con el contenido de flavonoides en las brácteas de $B$. glabra se obtuvo un coeficiente de correlación de $r=0,995$ (Tabla 2), denotándose que existe una correlación positiva fuerte, pues el análisis nos indica que la cantidad de fenoles totales y flavonoides están fuertemente correlacionados.

Del análisis de correlación entre fenoles vs. actividad antioxidante $\left(\mathrm{Cl}_{50}\right)$ y flavonoides vs. actividad antioxidante $\left(\mathrm{Cl}_{50}\right)$ se obtuvieron coeficientes de correlación $r=-0,999$ y 
$r=-0,994$, respectivamente, (Tabla 2)

denotando una correlación negativa en ambos casos; esto indica que en la medida que aumenta la cantidad de fenoles y flavonoides, disminuye el $\mathrm{Cl}_{50}$.
Tabla 2. Correlación estadística entre fenoles totales, flavonoides y actividad antioxidante

\begin{tabular}{lc}
\hline Correlación & $\mathbf{R}$ \\
\hline Fenoles vs. flavonoides & 0,995 \\
\hline $\begin{array}{l}\text { Fenoles vs. } \\
\text { actividad antioxidante }\end{array}$ & $-0,999$ \\
\hline $\begin{array}{l}\text { Flavonoides vs. } \\
\text { actividad antioxidante }\end{array}$ & $-0,994$ \\
\hline
\end{tabular}

\section{DISCUSIÓN}

Hay pocos estudios realizados en la cuantificación de fenoles totales en las brácteas de las variedades morada y naranja de $B$. glabra y, partiendo de un extracto hidroalcohólico, según lo reportado por Orozco et al. (2018), para las brácteas moradas de la especie Bougainvillea spectabilis el contenido de fenoles fue 6,78 mg EAG/g ES.

El método de extracción asistida por ultrasonido, utilizado para la obtención de los extractos hidroalcohólicos, resulta de gran efectividad, ya que genera mayor interacción entre el disolvente y la droga. Colateralmente, las ondas cavitacionales producidas por el equipo provocan la ruptura de las células, lo que favorece en el rendimiento de extracción de los metabolitos secundarios (Vinatoru, 2001).

En otro estudio realizado por Villanueva et al. (2017) determinaron la concentración de fenoles en extractos etanólicos al $50 \%$ de flores y brácteas de variedad rosada de $B$. xbuttiana, obteniendo 320 mg EAG/g ES. Dichos resultados son superiores a lo obtenido en esta investigación, tanto para la variedad naranja como para la variedad morada.

Con estos valores se puede apreciar que el contenido de fenoles en las brácteas de la especie $B$. glabra fue mayor a otras especies del mismo género, pero fue menor a la B. xbuttiana. 
El método más empleado comúnmente para cuantificar flavonoides se basa en la determinación espectrofotométrica del complejo (flavonoide$\mathrm{AlCl}_{3}$ ) que se forma entre los hidroxilos catecólicos (posición 3 y 5 del anillo $\mathrm{A} u$ otras variantes tanto en el anillo A como en el B) o del hidroxilo en 5 con el grupo carbonilo 4. Esto sucede principalmente en flavonoides del tipo flavona y flavonoles, el cual proporciona un desplazamiento batocrómico y un efecto hipercrómico. Este método es usado comúnmente para cuantificar flavonoides del tipo O-glicósidos sin descartar que la técnica ha sido utilizada indiscriminadamente para cuantificar flavonoides del tipo C-glicósidos (Dantas et al., 2012; Ramos et al., 2017).

Al comparar el contenido de flavonoides en este estudio con lo reportado por Markandan et al. (2016), ellos obtuvieron valores menores (2,49 mg EQ/g ES) en las brácteas moradas B. glabra tomadas de un jardín en Malasia. Esto puede deberse a que utilizaron metanol al $50 \%$ como disolvente y la extracción no fue asistida por ultrasonido. Además, es preciso considerar que los factores extrínsecos (clima, suelo y tempera- tura) e intrínsecos de la planta (estado fenológico) fueron diferentes.

A pesar de los múltiples usos farmacológicos de la B. glabra hay poca información sobre la actividad antioxidante de las brácteas de las variedades naranja y morada, procesadas de la forma antes descrita. Islam et al. (2016) y Shalini et al. (2018) evaluaron la capacidad antioxidante mediante el método de DPPH de las brácteas de $B$. glabra (morada) en un extracto acuoso y etanólico, obteniendo el $\mathrm{Cl}_{50}$ a $135,73 \mu \mathrm{g} / \mathrm{mL}$ y 75 $\mu \mathrm{g} / \mathrm{mL}$ respectivamente, valores menores que los obtenidos en las brácteas moradas analizadas en esta investigación.

Los resultados de correlación negativa, significativamente alta, entre flavonoides y fenoles $y$ actividad antioxidante (Tabla 2) infieren que la capacidad antioxidante puede atribuirse a estos metabolitos secundarios. Por lo que las muestras con mayores contenidos de fenoles totales y flavonoides requieren de menores concentraciones de los extractos de las brácteas de B. glabra, de colores morado y naranja, para inhibir el $50 \%$ del radical libre DPPH. 


\section{CONCLUSIÓN}

Este es el primer estudio en el cual se compara la relación existente entre el contenido de fenoles totales, flavonoides y la capacidad antioxidante en las brácteas naranjas y moradas de B. glabra. Las muestras de brácteas color naranja tuvieron las mayores concentraciones de compuestos fenólicos y de flavonoides. Adicionalmente, las dos muestras de brácteas (morada y naranja) pueden ser utilizadas como antioxidantes naturales, siendo la de variedad naranja la más activa.

\section{AGRADECIMIENTO}

A la Universidad Técnica de Machala de la Provincia de El Oro, Ecuador, a través del departamento de Investigación, por su patrocinio financiero, mediante el proyecto semillero LOBOCOM.

\section{LISTA DE REFERENCIAS}

Abarca, R. \& Petricevich, V. (2018). Bougainvillea genus: A review on phytochemistry, pharmacology, and toxicology. Evidence Based Complement. Alternative Medicine, 2018. 9070927. doi: 10.1155/2018/9070927

Alkadi, H. \& Ifeanyi, O.E. (2018) A Review on Free Radicals and Antioxidants. International Journal of Current Research in Medical Sciences, 4(2):123-33. doi:10.22192/ijcrms.2018.04.02.019.

Alonso-Castro, A. J., Domínguez, F., Maldonado-Miranda, J. J., Castillo-Pérez, L. J., Carranza-Álvarez, C., Solano, E., \& Ruiz-Padilla, A. J. (2017). Use of medicinal plants by health professionals in Mexico. Journal of Ethnopharmacology, 198, 81-86. 
Brand-Williams, W., Cuvelier, M.E. \& Berset, C. (1995). Use of a free radical method to evaluate antioxidant activity. Lebenson Wiss Technol. 28(1):25-30.

Cenini, G., Lloret, A. \& Cascella, R. (2019). Oxidative stress in neurodegenerative diseases: from a mitochondrial point of view. Oxidative Medicine and Cellular longevity. 2019,2105607. doi: 10.1155/2019/2105607.

Coronado, M., Vega, S., Gutiérrez, R., Vázquez, M., \& Radilla, C. (2015). Antioxidantes: perspectiva actual para la salud humana. Revista chilena de nutrición, 42(2), 206212. https://dx.doi.org/10.4067/S0717-75182015000200014.

Dantas, A. J., Assunção, M. R., Perrelli, K., Pereira, T., \& Lira, L. A. (2012). Total flavonoids content in the raw material and aqueous extractives from Bauhinia monandra Kurz (Caesalpiniaceae). Science World Journal, 2012. https://doi.org/10.1007/BF00 796455.

Enciso-Diaz, O. J., Mendez-Gutierrez, A., De Jesus, L. H., Sharma, A., Villarreal, M. L., \& Taketa, A. C. (2012). Antibacterial activity of Bougainvillea glabra, Eucalyptus globulus, Gnaphalium attenuatum, and propolis collected in Mexico. Pharmacology \& Pharmacy, 3(04), 433-438.

Heuer, S., Richter, S., Metzger, J. W., Wray, V., Nimtzt, M., \& Strack, D. (1994). Betacyanins from bracts of Bougainvillea glabra. Phytochemistry, 37(3), 761-767.

Islam, M. Z., Hossain, M. T., Hossen, F., Akter, M. S. \& Mokammel, M. A. In-Vitro antioxidant and antimicrobial activity of Bougainvillea glabra flower. Research Journal of Medicinal Plant, 10(3), 228-236. https://doi.org/10.3923/rjmp.2016.228.236.

Jamshidi-Kia, F., Wibowo, J. P., Elachouri, M., Masumi, R., Salehifard-Jouneghani, A., Abolhassanzadeh, Z., \& Lorigooini, Z. (2020). Battle between plants as antioxidants with free radicals in human body. Journal of Herbmed Pharmacology, 9(3), 191-199.

Lin, J. Y., \& Tang, C. Y. (2007). Determination of total phenolic and flavonoid contents in selected fruits and vegetables, as well as their stimulatory effects on mouse splenocyte proliferation. Food chemistry, 101(1), 140-147. https://doi.org/10.1016/j. foodchem.2006.01.014. 
Markandan, S., Abdullah, A., Musa, K. H., Subramaniam, V., \& Stockham, K. (2016). Determination of antioxidant activities, total phenolic and flavonoid contents in Bougainvillea glabra bracts at various methanol concentrations. In AIP Conference Proceedings, https://doi.org/10.1063/1.4966776.

Naidu, N., Srilekhs, R., Pethuru, V., Anusha, B., Kejiya, E., \& Lakshmamma, E. B. (2016). Evolution of anti-microbial and antioxidant activity of alcoholic extract of Bougainvillea glabra flowers. Indo American Journal of Pharmaceutical Research, 2, 67-71.

Orozco, J., Escobar, A., Buendía, L., García, C., Hernández, C., \& Álvarez, J. (2018). Evaluation of the protection and release rate of Bougainvillea (Bougainvillea spectabilis) extracts encapsulated in alginate beads. Journal of Dispersion Science and Technology. https://doi.org/10.1080/01932691.2018.1496834.

Ramos, R. T. M., Bezerra, I. C. F., Ferreira, M. R. A., \& Lira, L. A. (2017). Dual Anti-cholinesterase activity of Ajoene by in silico and In Vitro studies. Pharmacognosy Research, 9(3), 253-260. https://doi.org/10.4103/pr.pr.

Rao, K. B., Nidhi, H., Dipankar, D., Garima, D., Kumar, G., \& Karthik, L. (2015). Phytochemical profile, in vitro antioxidant property and HPTLC analysis of methanol extract of Bougainvillea glabra (Nyctaginaceae). International Journal of Pharmaceutical Sciences Review and Research, 31(2), 235-241.

Reyes, A., Galicia, M. \& Carrillo, M. (2011). Antioxidantes: la magia de lo natural. Revista Tlatemoani, (8), 1-16.

Saleem, H., Usman, A., Mahomoodally, M. F., \& Ahemad, N. (2021). Bougainvillea glabra (Choisy): A comprehensive review on botany, traditional uses, phytochemistry, pharmacology and toxicity. Journal of ethnopharmacology, 113356. https://doi.org/ 10.1016/j.jep.2020.113356.

Shalini, M., Aminah, A., Khalid, H. M., Vimala, S., Katherine, S., \& Khoo, M. G. H. (2018). In vitro antioxidant activities, phytoconstituent and toxicity evaluation of local Bougainvillea glabra bract (Bunga Kertas). International Journal of ChemTech Research, 11(09), 22-30. 
Villanueva, R. G., Abarca, R. V. \& Petricevich, V. L. (2017). Chemical compounds and biological activity of an extract from Bougainvillea xbuttiana (Var. Rose) Holttum and Standl. International Journal of Pharmacy and Pharmaceutical Sciences, 9 (3), 42.

Vinatoru, M. (2001). An overview of the ultrasonically assisted extraction of bioactive principles from herbs. Ultrasonics sonochemistry 8(3), 303-313. https://doi.org/10. 1016/S1350-4177(01)00071-2.

World Health Organization. (2013). WHO traditional medicine strategy: 2014-2023. World Health Organization. 